tion of the Chandler term. The presence of a beat would not definitely establish the correctness of our model, but would provide strong evidence in support of the basic idea. Even if the beat were absent, and the apparent change in amplitude illusory, it is still quite conceivable that the excitation of the Chandler motion could take place through the differential luni-solar gravitational torques acting, say, on the core and mantle which may interact through a non-linear coupling.

The presence of two important frequency components in the Chandler motion is, of course, also consistent with the apparent breadth of the power spectrum of the polar motion in the vicinity of $0 \cdot 8$ cycles $/ \mathrm{yr}^{1}$. In the past, the corresponding decrease in the amplitude of the auto correlation of the Chandler data-approxımately 10 per cent for a 14 month lag-was interpreted as indicating a damping time of approximately a decade and led to suggestions that the free nutation was being excited by random impulses ${ }^{1}$. In our model, the amplitude of the auto correlation will also decrease, as observed, but after longer intervals will rise again, the period being equal to that of the beat frequency. Furthermore, the problem of the anomalously small $Q$ value for the Earth, inferred from the "damping" of the Chandler motion, is automatically disposed of.

If this model of the Chandler wobble proves fruitful, it might be interesting to consider the more complicated case of a non-axially symmetric mantle which may be appropriate for Venus or the Moon.

\section{G. Colombo}

Smithsonian Astrophysical Observatory,

Cambridge, Massachusetts, and

University of Padua,

Italy.

\section{I. Shapiro}

Department of Geology and Geophysies and

Department of Physics,

Massachusetts Institute of Technology.

Received Oetober 9; revised November 9, 1967.

${ }^{1}$ See, for example, Munk, W. H., and MacDonald, G. J. F., in The Rotation of the Earth (Cambridge Univ. Press, London, 1960).

${ }^{2}$ Chandler, S. C., Astron. J., 11, 65 (1891)

${ }^{3}$ Newenmb. S.. Astron. J.. 11, 81 (1891).

\section{PHYSICS}

\section{Dimensional Problem of the Power Law in Rheology}

THE power law equation, between shear stress, and strain rate, $\dot{\gamma}$, may be written $\dot{\gamma}=k \tau^{a}$ : it has often been shown $n^{1,2}$ to be in accordance with experimental data obtained for many non-Newtonian fluids. The equation is open to the logical objection that it is dimensionally inconsistent unless the constant of proportionality, $k$, is not a true constant at all but has variable dimensions which depend on the properties of the sample under observation. Nedonchelle and Schutz $^{3}$ have shown experimentally that for one particular non-Newtonian system there is a relationship between the constant of proportionality, $k$, and the exponent, $\alpha$, such that the equation may be reduced to a dimensionless form containing three dimensionally stable constants which are inde. pendent properties characterizing the material in its environment.

Scott Blair ${ }^{4}$ attempted to provide a theoretical basis for the existence of the power law, and in one form ${ }^{5}$ of his derivation he introduced four constants, two of which were particular values of a function, which he designated $f$ in his paper. In his treatment, this function was subsequently eliminated from the argument, but it can easily be shown that the constants of Nedonchelle and Schutz and of the particular values of Scott Blair for $f$ are closely related.

Writing the dimensionless equation of Nedonchelle and Schutz in the form

$$
\frac{\dot{\gamma}}{\dot{\gamma}_{0}}=\left(\frac{\tau}{\tau_{0}}\right)^{a}
$$

to correspond with the form given here, by replacing their $D$ by $\dot{\gamma}$ and their $a$ by $1 / \alpha$, gives, on taking logarithms

$$
\log \dot{\gamma}-\log \dot{\gamma}_{0}=\alpha \log \tau-\alpha \log \tau_{0}
$$

From the theoretical derivation of Scott Blair, $\alpha$ is the ratio of two constants, which he denotes by $a$ and $b$. Equation (2) may then be rewritten

$$
b \log \dot{\boldsymbol{\gamma}}-b \log \dot{\boldsymbol{\gamma}}_{0}=a \log \tau-a \log \tau_{0}
$$

or

$$
a \log \tau-b \log \gamma=a \log \tau_{0}-b \log \dot{\gamma}_{0}
$$

Scott Blair gives (his equations (2) and (4))

so that

$$
a \log \tau=f_{1}-f \quad b \log \dot{\gamma}=f_{2}-f
$$

$$
a \log \tau-b \log \dot{\boldsymbol{\gamma}}=f_{1}-f_{2}
$$

Combining equations (3) and (4) gives

$$
\alpha \log \tau_{0}-b \log \gamma_{0}=f_{1}-f_{2}
$$

This becomes an identity if

$$
f_{1} \equiv a \log \tau_{0} \quad f_{2} \equiv b \log \dot{\gamma}_{0}
$$

These expressions (5) give the relaticn between the experimentally determinable constants $\tau_{0}$ and $\dot{\gamma}_{0}$ of Nedonchelle and Schutz, and the constants of integration $f_{1}$ and $f_{2}$ of Scott Blair.

Thus from the results of Nedonchelle and Schutz, it can be deduced that a minimum of three independent characteristic constants are necessary and sufficient for the power law to be dimensionally acceptable, and these three constants are compatible with the theoretical derivation of Scott Blair.

\section{J. H. Prentice}

National Institute for Research in Dairying, Shinfield, Reading.

Received November 21; revised December 13, 1967.

' Philippoff, W., in Viskosität der Kolloide (Dresden, 1942).

2 Spriggs, T. W., Huppler, J. D., and Bird, R. B., Trans. Soc. Rheol., 10, 191 (1966).

${ }^{3}$ Nedonchelle, Y., and Schutz, R. A., CR Acad. Sci.,265 C, 16 (1967).

4 Scott Blair, G. W., Rheologica Acta, 4, 53 (1965).

'Scott Blair, G. W., and Prentice, J. H., Cah. du Gpe. Fr, de Rhécl, 2, 75 (1966).

\section{Luminescent Properties of Rocks, Meteorites and Natural Glasses under Proton Bombardment}

SUGGESTIONS that some transient lunar events ${ }^{1}$ are luminescent phenomena, excited by solar protons, have aroused interest in the luminescent properties of putative lunar surface materials. We have studied the luminescence produced in various minerals and meteorites by a beam of $146 \mathrm{MeV}$ protons from the Atomic Energy Research Establishment synchrocyclotron. Both our proton flux, about $10^{8}$ protons $\mathrm{sec}^{-1} \mathrm{~cm}^{-2}$, and the ionization density are many orders of magnitude lower than those used by other workers ${ }^{2-4}$; thus we avoid their problems of radiation damage and simulate more closely the conditions on the Moon's surface. 